\section{Structural Imaging Reveals Anatomical Alterations in Inferotemporal Cortex in Congenital Prosopagnosia}

Marlene Behrmann ${ }^{1}$, Galia Avidan ${ }^{2}$, Fuqiang $\mathrm{Gao}^{3}$ and Sandra Black $^{3}$

${ }^{1}$ Department of Psychology, Carnegie Mellon University, Pittsburgh, PA 15213-3890, USA, ${ }^{2}$ Tel Aviv Sourassky Medical Imaging Center, Tel Aviv, Israel and ${ }^{3}$ Cognitive Neurology, Sunnybrook Health Science Center, University of Toronto, Toronto, Canada

\begin{abstract}
Congenital prosopagnosia (CP) refers to the lifelong impairment in face recognition in individuals who have intact low-level visual processing, normal cognitive abilities, and no known neurological disorder. Although the face recognition impairment is profound and debilitating, its neural basis remains elusive. To investigate this, we conducted detailed morphometric and volumetric analyses of the occipitotemporal (OT) cortex in a group of CP individuals and matched control subjects using high-spatial resolution magnetic resonance imaging. Although there were no significant group differences in the depth or deviation from the midline of the OT or collateral sulci, the CP individuals evince a larger anterior and posterior middle temporal gyrus and a significantly smaller anterior fusiform (aF) gyrus. Interestingly, this volumetric reduction in the aF gyrus is correlated with the behavioral decrement in face recognition. These findings implicate a specific cortical structure as the neural basis of CP and, in light of the familial history of CP, target the aF gyrus as a potential site for further, focused genetic investigation.
\end{abstract}

Keywords: congenital prosopagnosia, cortex structure, face processing, human brain anatomy, neuropsychology, ventral visual cortex

\section{Introduction}

Congenital prosopagnosia (CP) is a lifelong debilitating impairment in face processing that occurs despite intact visual, social, and intellectual functions (Nunn et al. 2001; Kress and Daum 2003a; Behrmann and Avidan 2005; Duchaine and Nakayama 2006; Duchaine et al. 2006). Individuals with CP fail to recognize people, even close family members, and exploit cues such as an individual's voice or clothing or accessories to assist them with recognition. The deficit extends beyond recognition per se, however, as $\mathrm{CP}$ individuals are also impaired, relative to control subjects, in making perceptual discriminations between 2 unknown faces (Bentin et al. 1999; Behrmann and Avidan 2005; Behrmann et al. 2005; Yovel and Duchaine 2006). As a means of coping with the disorder, some individuals with CP have developed particular strategies such as greeting all whom they encounter or, alternatively, as being somewhat socially reticent.

Although CP parallels the characteristics of acquired prosopagnosia (AP) and can be as severely debilitating (Behrmann et al. 2005; Le Grand et al. 2006), the neural basis of AP is wellestablished, whereas the neural origin of $\mathrm{CP}$ remains elusive. AP typically occurs in individuals following a lesion (De Renzi 1986; Sergent and Signoret 1992; De Renzi et al. 1994; Farah 2004) such as a bilateral or unilateral right hemisphere stroke in the inferior occipitotemporal (OT) cortex (De Renzi 1986; Sergent and Signoret 1992; De Renzi et al. 1994; Farah 2004; Kleinschmidt and Cohen 2006), and classically implicates the anterior tem- poral lobe and the fusiform and lingual gyri (Damasio and Damasio 1980; Damasio et al. 1986; Barton 2003; Barton et al. 2004; Bouvier and Engel 2006). In contrast, CP occurs in the absence of any obvious discernible lesion, examined using conventional neuroimaging, or any other neurological concomitant (Jones and Tranel 2001; Kress and Daum 2003a; Duchaine and Nakayama 2006). Until recently, CP was thought to be rare but the increase in reports of this disorder suggests that it may not be that uncommon (Kress and Daum 2003a; Behrmann and Avidan 2005; Behrmann et al. 2005; de Gelder and Stekelenburg 2005; Duchaine and Nakayama 2005; Duchaine and Nakayama 2006). Documenting the neural basis of this neurodevelopmental disorder may elucidate not only the mechanisms underlying CP itself, and face recognition more generally, but may also serve as a model for other similar neurodevelopmental disorders such as developmental dyslexia or congenital amusia. Additionally, because many of these CP individuals also have affected family members, implicating a genetic basis for the disorder (De Haan 1999; Grueter et al. 2005; Kennerknecht et al. 2006), identifying the neural correlate of this disorder will specify cortical sites for targeted genetic investigation and can potentially shed light on the architectural specification of ventral temporal cortex.

Given that the injury to the inferior OT cortex is the most frequent neuroanatomical substrate in $\mathrm{AP}$, we took it to be a reasonable candidate as the basis of $\mathrm{CP}$. To date, however, neither functional magnetic resonance imaging (fMRI) studies nor physiological measures such as evoked response potential (ERP) or magnetoencephalography (MEG) have succeeded in documenting definitive changes in this cortical region in all cases of CP. Although some fMRI investigations of CP individuals (Hadjikhani and De Gelder 2002; Duchaine et al. 2006) have revealed abnormal profiles in the "fusiform face area" (FFA), the preeminent face processing area (Kanwisher et al. 1997), other detailed fMRI studies in CP report normal face-related activation in ventral visual cortex even in the FFA itself (Hasson et al. 2003; Avidan et al. 2005; von Kriegstein et al. 2006). Surprisingly, too, CP individuals show normal face- and object-selective blood oxygen level-dependent (BOLD) adaptation levels in inferotemporal cortex and, like control subjects, exhibit evidence of global representation of faces in the FFA as reflected in FFA activation during the perception of faces but not vases in response to an ambiguous face/vase stimulus (Avidan et al. 2005). Additionally, in ERP and MEG studies, a conspicuous $\mathrm{N} 170$ or M170 is detectable in some, but not in all, individuals with CP (Bentin and Deouell 2000; Kress and Daum 2003b; Harris et al. 2005), although it may also be elicited for objects, suggesting a possible lack of specificity for faces in this early waveform. Surprisingly, conventional structural MRI reveals no 
observable impairment (Jones and Tranel 2001), although one case study that suggests that the $\mathrm{CP}$ individual under investigation, YT, probably had a smaller right temporal lobe than the matched control subjects (Bentin et al. 1999).

To explore the neural basis of $\mathrm{CP}$, we undertook detailed structural and anatomical analyses of the inferior OT cortex in a group of individuals with $\mathrm{CP}$ and matched control participants. We conducted sulcal tracing and sulcal depth and deviation analyses, as well as parcellation and volumetric assessment of the temporal lobe, using procedures that have been used successfully with other neurodevelopmental populations in whom no obvious cortical lesion is evident. Using such procedures, reduced overall temporal lobe volumes, especially involving the middle and inferior temporal gyri have been observed in individuals with developmental dyslexia, with greater reduction in the left than right temporal lobe, as might be predicted (Eliez et al. 2000; Brown et al. 2001; Casanova et al. 2005; Vinckenbosch et al. 2005). Using similar procedures, anatomical alterations have also been documented in individuals with developmental dyscalculia (difficulties in numerical representation) showing that their right intraparietal sulcus differs in length, depth, and geometry relative to their counterpart controls (Molko et al. 2003). There are also many neurostructural studies conducted in individuals with Williams' syndrome (WS) (Reiss et al. 2004) in which, for example, reduced thalamic and parietal and occipital lobe volumes are noted, perhaps accounting for the marked impairment in the visuospatial domain. There are, of course, many studies documenting cortical changes associated with autism (for recent review, see Levitt et al. 2003; Belmonte et al. 2004), schizophrenia (Lee et al. 2002; Vidal et al. 2006), and aging (Rettmann et al. 2005). CP, however, unlike these latter cases, and more akin to dyslexia, dyscalculia, and WS, has a more restricted cognitive decrement and the goal here is to explore the neural substrate associated with this more limited cognitive perturbation and to shed light on the neurostructural profile of the candidate region for $\mathrm{CP}$, the inferotemporal cortex. The advent of advanced noninvasive high-resolution imaging procedures now allows unprecedented opportunities to demarcate ana- tomical alterations in vivo in humans and to identify a structural neural marker that may aid behavioral analyses in studying genotype-phenotype relations.

\section{Materials and Methods}

\section{Subjects}

Six healthy CP subjects ( 3 males) aged between 29 and 73 years, with normal vision, no discernable cortical lesion, and no history of any neurological or psychiatric disease participated in all behavioral and anatomical experiments. Twelve age-, handedness-, and gendermatched individuals ( 2 per each CP individual) with no neurological or psychiatric history served as control subjects. All participants were right-handed, as established on the Edinburgh handedness inventory (Oldfield 1971), had normal or corrected-to-normal vision, and intact low-level visual processing.

All subjects consented to participate in the behavioral and imaging experiments, and the protocol was approved by the Institutional Review Boards of Carnegie Mellon University and of the University of Pittsburgh. All CP individuals have undergone extensive testing to verify the diagnosis of CP. Behavioral and functional imaging data from the first 4 of the $6 \mathrm{CP}$ subjects have been published previously and the reader is also referred to those papers for additional details (Avidan et al. 2005; Behrmann and Avidan 2005; Behrmann et al. 2005). Data from the additional 2 subjects are included below.

\section{MRI Acquisition}

All subjects were scanned at the Brain Imaging Research Center, Pittsburgh, PA, on a 3-T Siemens Allegra scanner equipped with a standard head coil. High-resolution anatomical scans ( $T_{1}$-weighted 3D magnetization prepared rapid gradient echo) were acquired with the following parameters: time echo $=3.49$, flip angle $=8^{\circ}$, field of view $=$ $256 \times 256 \mathrm{~mm}^{2}$, matrix size $=256 \times 256$, slice thickness $=1 \mathrm{~mm}$, number of slices $=160-192$, orientation of slices was either horizontal or sagittal. All 3D- $T_{1}$ MR images were aligned to anterior-posterior commissure (AC-PC) plane with head-tilt corrections in all 3 orientations. The skull was stripped away using the BET program to obtain clean cortical images, and the cerebellum and brainstem were then manually "removed" to expose collateral (C) and OT sulci on the inferior surface of the brain (see Fig. 1A).

\section{Sulcal Analysis}

For each $\mathrm{CP}$ and control individual, the OT and $\mathrm{C}$ sulci were manually traced on the inferior surface (see Fig. $1 A, B$ ). To superimpose the
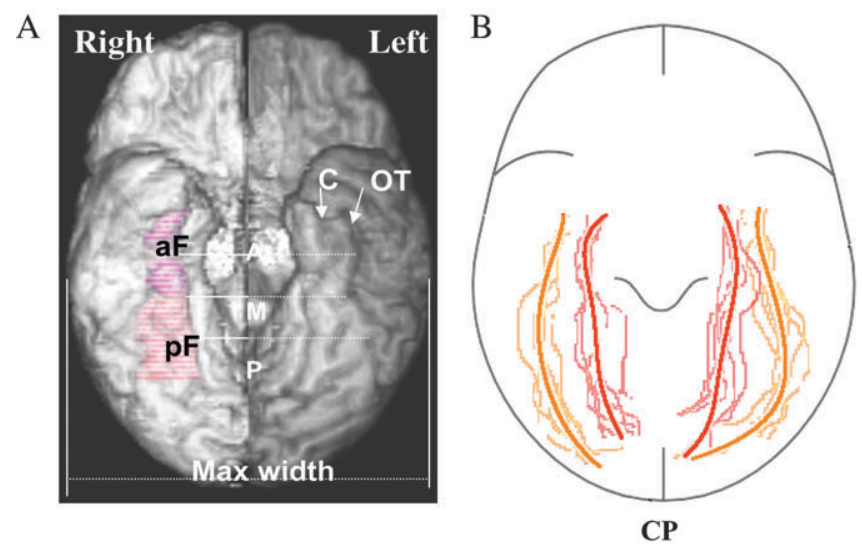
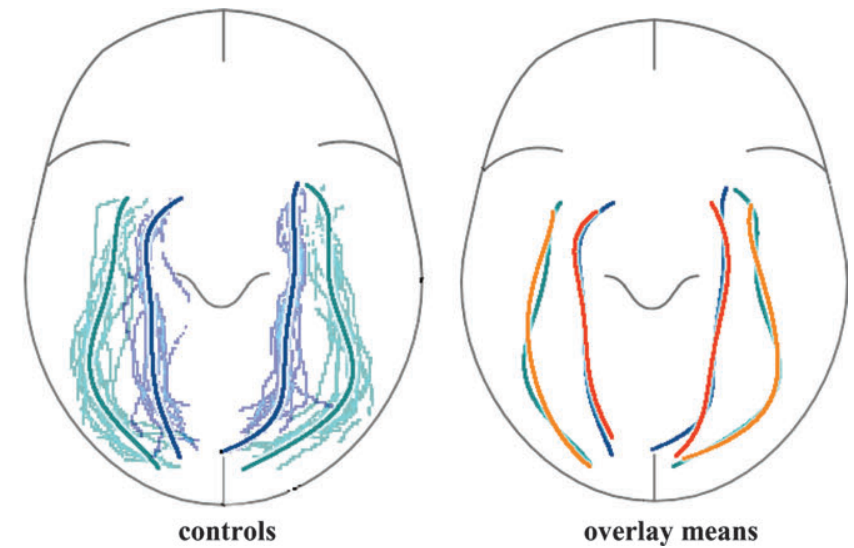

Figure 1. Tracing of inferior temporal sulci for $\mathrm{CP}$ and control participants and fusiform gyrus demarcation. (A) Ventral view of inferior surface of a normal brain with cerebellum and brainstem stripped away. Anterior (AF) and posterior (PF) fusiform tracings in the right (R) hemisphere, and tracing of the $\mathrm{C}$ and OT sulci in the left (L) hemisphere. Deviation of the $\mathrm{C}$ and OT sulci from the midline was measured at levels of A (midway of the aF), P (midway of the pF), and M (midway between the anterior and posterior end of the fusiform gyrus). The solid line indicates the distances between the midline and $\mathrm{C}$ and the dashed line between the midline and OT. The maximal width from side to side was measured to correct for differences in head width. $(B)$ Individual tracings for $C$ and OT sulci for each of $6 \mathrm{CPs}$ (left panel: orange $=0 \mathrm{~T}$; red $=\mathrm{C}$ ) and each of 12 control participants (middle; green $=0 \mathrm{~T}$; blue $=\mathrm{C}$ ) and the overlay of the group means (right panel). Orange and green: OT sulcus. Red and blue: $\mathrm{C}$ sulcus. Thinner lines: each individual sulcus. Thicker lines: the trends of all individual lines in each group generated using least square technique. 
tracings so that the group differences could be explored, the scans were spatially normalized to the standard $T_{1}$-MRI template provided as part of SPM 99 (http://www.fil.ion.ucl.ac.uk/spm) and the spatial normalization protocol included a linear 12-parameter affine transformation (nonlinear transformations may alter the location of the sulci and were thus avoided). The sulcal patterns for each individual were then overlaid using MRIcro in the standardized template image for each hemisphere. Note that the tracings were all done by a trained research neuroradiologist (F.Q.) who was blind to the identity and performance profile of the individual participants.

To quantify and compare the sulcal morphometry across the $\mathrm{CP}$ and control groups, we measured both the distance of the C and OT sulci from the midline as well as the depth of the sulci (see Figs 1 and 2). The distance of the $\mathrm{C}$ and $\mathrm{OT}$ sulci in both hemispheres from the midline was measured at levels of the midpoint of the anterior fusiform (aF), the midpoint of the posterior fusiform $(\mathrm{pF})$, and midway between the anterior and posterior end of the fusiform gyrus. Maximal width from side to side was measured and then distance normalized (distance value divided by maximal width) to correct for the possible effect of headsize on any observable differences (see Fig. $1 A$ for illustration). The depth of the $\mathrm{C}$ and OT sulci was measured by a straight line between the deepest point and the surface opening of the sulcus (see Fig. 2). This depth measurement was taken on every fourth slice in the range of $\mathrm{aF}$ and $\mathrm{pF}$, and a correction for headsize (normalizing by entire intracranial area) was used.

\section{Volumetric Analysis}

For all participants, we quantified the volume of 9 different subregions of the temporal lobe, corresponding to the fusiform gyrus (subdivided into anterior and posterior), hippocampus, parahippocampus, superior temporal gyrus, and middle and inferior temporal gyrus (subdivided into anterior and posterior). These measurements were all done manually in an attempt to be maximally conservative as automatic parcellation procedures work optimally with normal brains but it is not clear whether these procedures work with brains that are anatomically atypical (and the point of the current exploration is to determine this very issue). The planimetric tracings were carried out blind by an experienced observer (F.Q.) who has conducted similar previous studies. The procedure laid out by Lee et al. (2002) in their analysis of the fusiform gyrus in schizophrenia was adopted and followed rigorously to obtain these measurements.

First, the cortex of the fusiform gyrus was traced on coronal slices perpendicular to the AC-PC line (Fig. 3). The medial and lateral boundaries of the fusiform gyrus were defined using the published protocol of Lee et al. (2002): fusiform cortex was traced starting at the

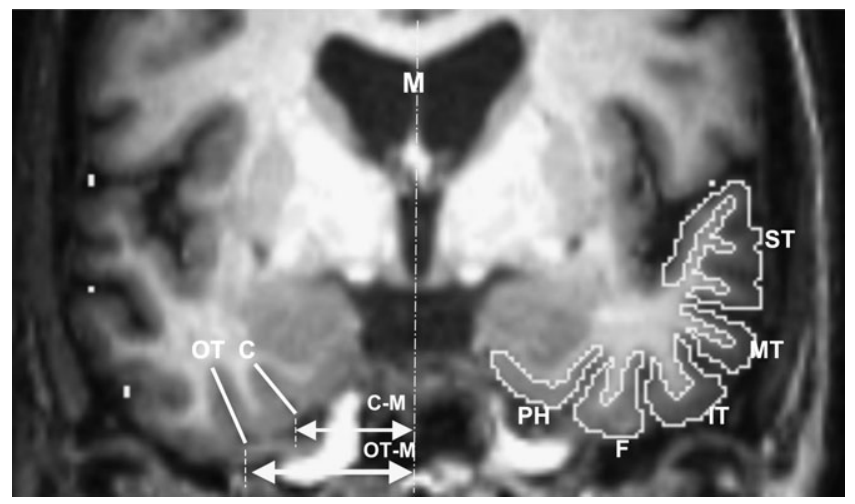

Figure 2. Inferior temporal sulcal depth and deviation analysis. The depth of the $\mathrm{C}$ and OT sulci is measured in the coronal sections by a straight line between the deepest point and the surface opening of the sulci. The depth is measured at every fourth slice in the range of whole $\mathrm{aF}$ and $\mathrm{pF}$ gyri. Deviations of the $\mathrm{C}$ sulcus from the midline $(\mathrm{C}-\mathrm{M})$ and OT sulcal from the midline (OT-M) in each hemisphere are measured at levels of the midpoint of the $\mathrm{aF}$, the midpoint of the $\mathrm{pF}$, and the midpoint between the anterior and posterior end of the fusiform. This is shown on the left side of the picture. On the right is an illustration of tracing of 5 temporal subregions: $\mathrm{PH}=$ parahippocampus, $\mathrm{F}=$ fusiform, IT = inferior temporal, and ST = superior temporal. first slice posterior to the pituitary stalk (see Fig. 3A) and ending at the slice midway between the posterior commissure and the posterior end of the occipital lobe at the AC-PC level (see Fig. 3B). The $\mathrm{C}$ sulcus was used as the medial border and the OT sulcus was used as the lateral border (Fig. 3C). The fusiform gyrus was divided into anterior and posterior portions at the midpoint between the anterior and posterior ends of the tracing (Fig. 3C). Parahippocampal and hippocampal volumes were also obtained based on a well-established protocol (Callen et al. 2001): the entire hippocampus was traced on the sagittal sections. The parahippocampal cortex was traced on coronal slices starting from the first slice posterior to the pituitary stalk and ending at the first slice showing the crus of the fornix. The volumes of the hippocampus, parahippocampal, and fusiform cortex were obtained by manual tracing on every other slice in the AC-PC aligned 3D- $T_{1}$ MRI using the ROI module of ANALYZE software (ANALYZE 2004).

The anterior end of the temporal gyri is the same as for the parahippocampal and hippocampal volumes (at the pituitary stalk) and the posterior end was defined by the appearance of the occipitoparietal sulcus (see Fig. 5). The superior temporal gyrus was demarcated, the gray matter traced and its volume calculated (see Fig. 5). The middle and inferior temporal gyri were divided into anterior and posterior components at the crux of the fornix (see Fig. $5 C$ ), which is also the end slice of the superior temporal gyrus and the parahippocampus.

For all subregions, because the tracing is done on alternate slices, the obtained volume is multiplied by 2 to yield the full complement. All the above calculations were done for each $\mathrm{CP}$ and each control subject individually and were also done separately for the left and right hemisphere. All values are headsize corrected using a published protocol in which the volume is divided by the size of the intracranial area and then multiplied by the intracranial area averaged across all participants (Laakso et al. 1996). Note that because the measures we are using have been adopted from existing protocols (and their reliability explored previously), we have not established the precision of these dependent measures independently but refer the reader to the original papers for further discussion of the reliability of the measures.

\section{Results}

Analysis of Bebavioral Impairment in the CP Individuals

All participants completed 3 behavioral tasks-many others were also completed (Behrmann et al. 2005; Avidan et al. in press; Behrmann et al. in press; Humphreys et al. in press) but these 3 suffice to characterize the face processing impairment for the current purposes. On the famous face recognition task, faces of well-known celebrities, such as Ronald Reagan and Bill Clinton, were presented individually for unlimited exposure duration until the participants provided a name for the face, provided any other relevant information about the face (e.g., profession), or said that they did not know anything about the face. All CP subjects' scores fell significantly below the distribution of the particular control participants in their ability to identity the famous faces (see Table 1 for results). The CP subjects also performed significantly more poorly than the controls on a face discrimination task in which participants were shown 2 novel faces for an unlimited exposure duration on a computer screen, decided whether the faces were the same or different, and pressed one of 2 keys to indicate their response. The $z$ scores for the CP participants, calculated based on the mean and range of the control reaction time (RT) data (accuracy is uniformly high given the extended exposure duration), are shown in Table 1. Again, all CP individuals fell below the normal distribution in this task. Although they performed poorly at face recognition and discrimination, the $\mathrm{CP}$ individuals were able to detect the presence of a face well: When required to press a key 

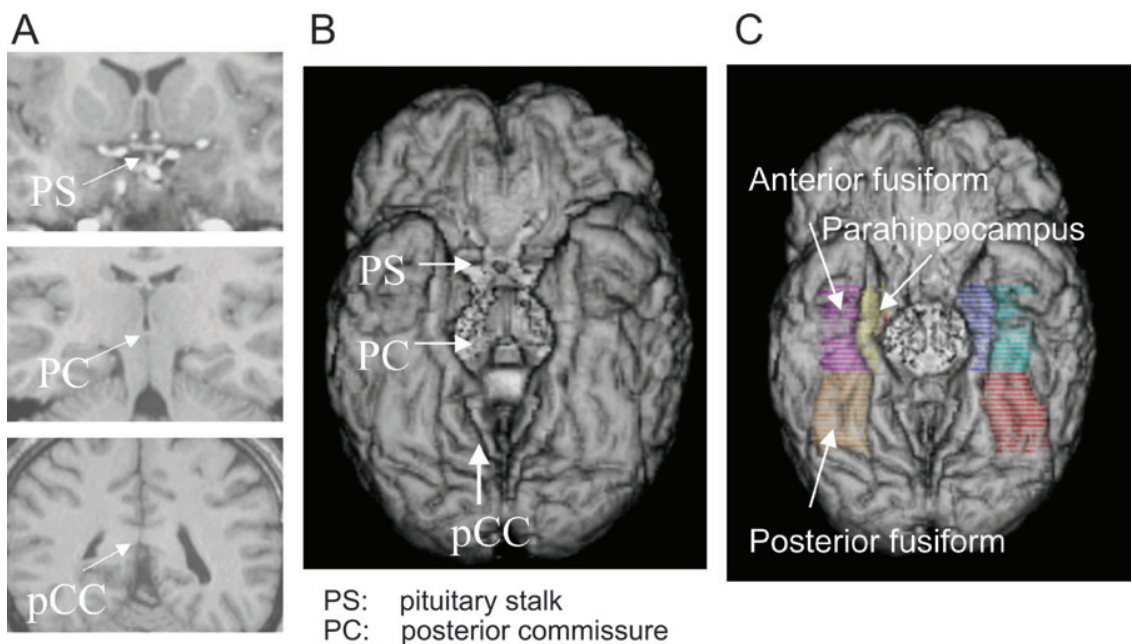

PS: pituitary stalk

PC: posterior commissure

pCC: posterior end of corpus collosum at midline

Figure 3. Protocol for temporal lobe subdivision. ( $A$ and $B$ ) Identification of midline structures including the pituitary stalk (PS), posterior commissure (PC), and posterior end of the corpus callosum (pCC). (C) The aF and parahippocampal gyrus are defined by the PS anteriorly and the PC posteriorly and the pF is defined by the PC anteriorly and the pCC posteriorly.

Table 1

Performance data for $6 \mathrm{CP}$ and control participants on 3 tests of face processing

\begin{tabular}{|c|c|c|c|c|c|}
\hline & & & $\begin{array}{l}\% \text { Face } \\
\text { recognition }\end{array}$ & $\begin{array}{l}\text { Face } \\
\text { discrimination (RT) }\end{array}$ & $\begin{array}{l}\% \text { Face } \\
\text { detection }^{\mathrm{a}}\end{array}$ \\
\hline Controls $(N=12)$ & & & 87 & $1598 \mathrm{~ms}$ & 94.3 \\
\hline $\mathrm{CP}$ participants & Sex & Age & & & \\
\hline KM & $\mathrm{F}$ & 60 & 41 & $P<0.001$ & 92 \\
\hline TM & $M$ & 27 & 47 & $P<0.01$ & - \\
\hline MT & $M$ & 41 & 46 & $P<0.0001$ & 100 \\
\hline $\mathrm{BE}$ & $\mathrm{F}$ & 28 & 11 & $P<0.0001$ & 92 \\
\hline IT & $\mathrm{F}$ & 73 & 21 & $P<0.001$ & 91 \\
\hline$M X$ & $M$ & 42 & 76 & $P<0.05$ & 100 \\
\hline
\end{tabular}

${ }^{a}$ We were unable to collect these data from TM for logistic reasons.

to indicate detection of a face or a car from amongst a stream of 250 rapidly presented input images, of which $10 \%$ were faces and $10 \%$ cars, the CP individuals all showed equivalently high accuracy for both face and car detection (see Table 1 for face detection data; face vs. cars accuracy, $F<1$, not significant [n.s.]). Further support for the intact detection in CP is that the average RT of the CP group for face and car detection was equivalently fast (faces: $563 \mathrm{~ms}$, cars: $596 \mathrm{~ms}$, n.s., and not significantly different from controls). All CP subjects clearly meet the criterion for the diagnosis of CP exhibiting a significant impairment in face processing and having no apparent neurological concomitant. Having established that the CP individuals meet the criterion for inclusion, we now go on to report the results of the anatomical analyses.

\section{Analysis of Sulcal Deviation and Sulcal Deptb}

The results of the sulcal tracing for the CP and control individuals are shown in Figure $1(B)$ and, as is evident by observation, there are no obvious group differences in the organization or length of the C or OT sulci (see Methods for further detail regarding the procedures and Appendix 1 for tables containing the data for each individual). The quantification of the distance of the sulci from the midline permits a systematic comparison of any sulcal deviations between the groups. An analysis of variance was conducted on the distance scores from the midline (corrected for headsize) of these 2 inferior ventral sulci with hemisphere (left, right) $\times$ sulcus $(C$, OT) $\times$ anterior-posterior location (anterior, mid, posterior) as within-subject variables and group as a between-subjects variable. Unsurprisingly, there was significantly greater distance from the midline for the OT than C sulcus, $F_{1,16}=1456.4, P<$ 0.0001. Also, as expected, there was significantly greater distance from the midline for the OT sulcus than for the $\mathrm{C}$ sulcus especially at the middle and posterior points (interaction between sulcus $\times$ anterior-posterior location $\left(F_{2,32}=73.4, P<\right.$ 0.0001). Most importantly, however, there were no statistically significant main effects or interactions involving group, all $P>$ 0.1 , indicating that the sulcal path and extent of deviation from the midline was equivalent in the 2 groups.

In an analysis of the depth of the 2 sulci (see Method for description of derivation of depth measure and Appendix 1 for data), as with the sulcal deviation, of prime importance is that there are no statistical effects involving the factor of group, all $P>0.05$. Across groups, there is greater sulcal depth in the anterior than posterior regions for both sulci $\left(F_{1,16}=31.7, P<\right.$ $0.0001)$, and greater depth overall in the OT than in C sulcus $\left(F_{1,16}=5.6, P<0.05\right)$, with disproportionately greater depth posteriorly on the left for the OT sulcus than for any other measurement. Taken together, the findings from the sulcal midline deviation analysis and sulcal depth measures indicate that there are no differences in sulcal morphometry and patterning for the $\mathrm{CP}$ compared with the control group.

\section{Analysis of Volumetric Measurements of Temporal Cortex}

The volumetric analysis involved a delineation and comparison of the volumes of 9 separate regions of the temporal lobe in the $\mathrm{CP}$ and control groups. The volumes were measured according to established protocols, where possible (see Methods and Figs 3-5). To correct the volume for possible differences in headsize, all measures for each individual were normalized 

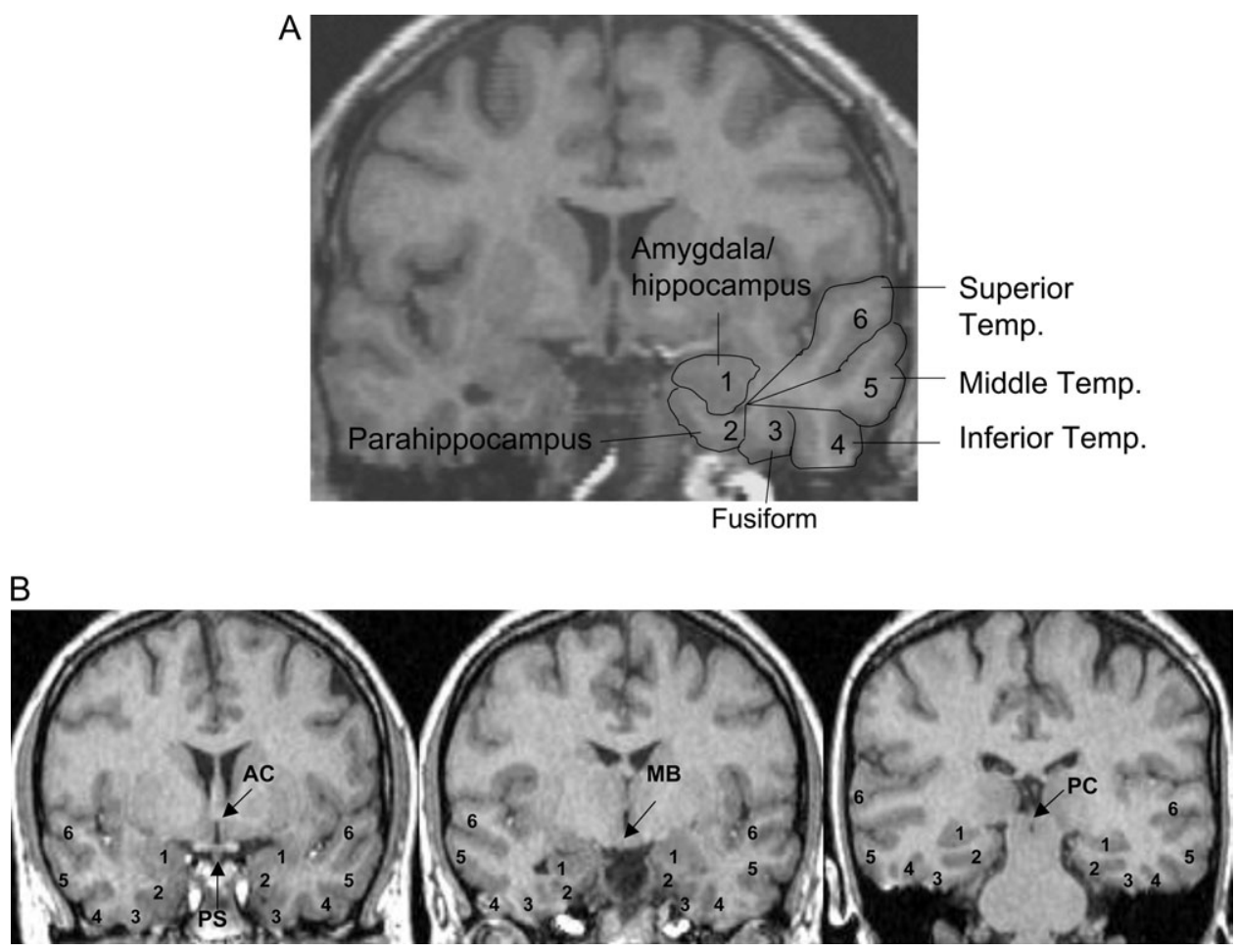

Figure 4. Demarcation of temporal lobe structures. (A) Coronal slice showing example of tracing the 6 temporal structures just anterior to the level of the pituitary stalk. (B) Coronal slices showing the tracing of the temporal structures at the level of the anterior commissure (AC) and pituitary stalk (PS), mammillary body (MB), and at the posterior commissure (PC).

A

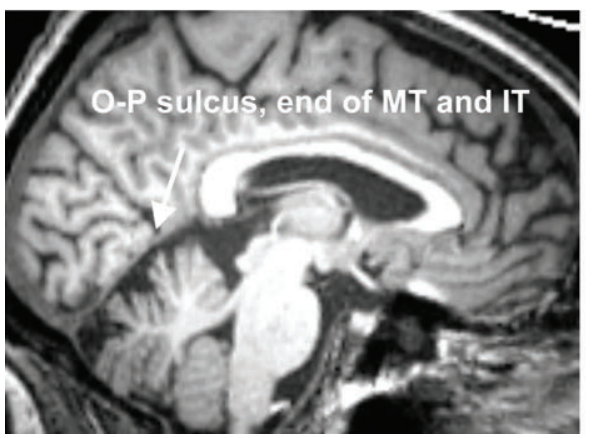

B

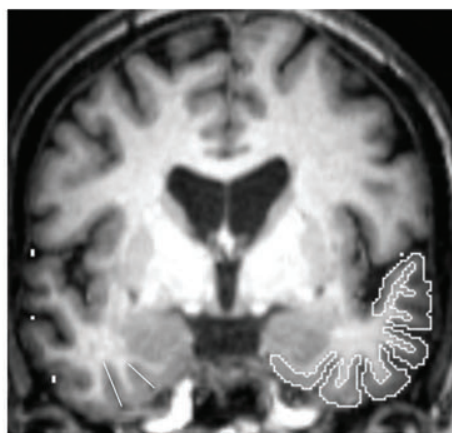

C

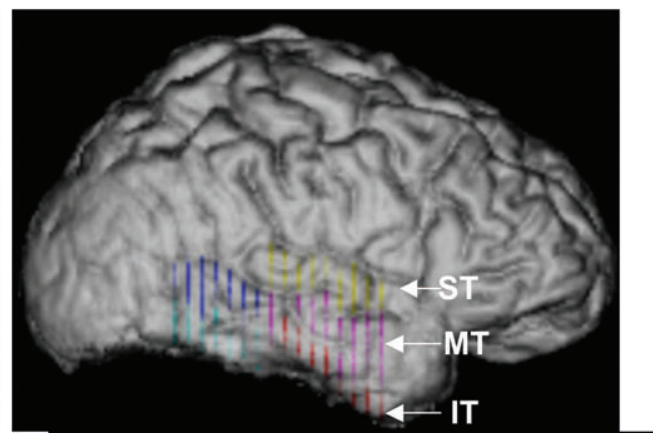

Figure 5. Definition and subdivision of the temporal gyrus. (A) Medial section showing the posterior end of the MT gyrus, defined by the appearance of the occipitoparietal sulcus $(0-P)$. (B) Coronal section showing tracing of cortical surface for the superior, middle, and inferior temporal gyri. $(C)$ Lateral view of the cortex showing separation into superior, middle, and inferior temporal gyri and the separation of the middle and inferior gyri into the more anterior versus posterior portions.

by total intracranial volume. Note that there was no significant difference in intracranial volume across the 2 groups (one-way analysis of variance $[\mathrm{ANOVA}]: F_{1,16}=1.3, P=0.25$ ). To compare the volumes of the regions of the temporal lobes across the 2 populations, we performed an ANOVA with group as a between-subjects measure and region $(N=9$; hippocampus, parahippocampus, aF, $\mathrm{pF}$, superior temporal gyrus, and anterior and posterior divisions of the middle and inferior temporal gyri) and side (left, right hemisphere) as within-subject factors.

The analysis reveals that there is no difference between the overall volumes for the right and left hemispheres, $P>0.1$, and that this left/right equivalence holds true for both groups (group $\times$ left/right volume: $P>0.2$ ). As might be expected, there is a significant difference in volume across the 9 different regions $\left(F_{8,128}=224.9, P<0.0001\right)$, collapsed across groups, with the superior temporal gyrus being the largest and the parahippocampus the smallest region (see Figs 4 and $6 A$ ). It is the case, however, that the regional sizes differed for the 2 hemispheres $\left(F_{8,128}=2.3, P<0.02\right)$ : whereas the superior temporal gyrus is significantly larger on the left than right (post hoc Tukey test: left 9257 vs. $8916 \mathrm{~mm}^{3}$ ), presumably related to language function and confirming many existing studies (Dorsaint-Pierre et al. 2006), the anterior and posterior middle 

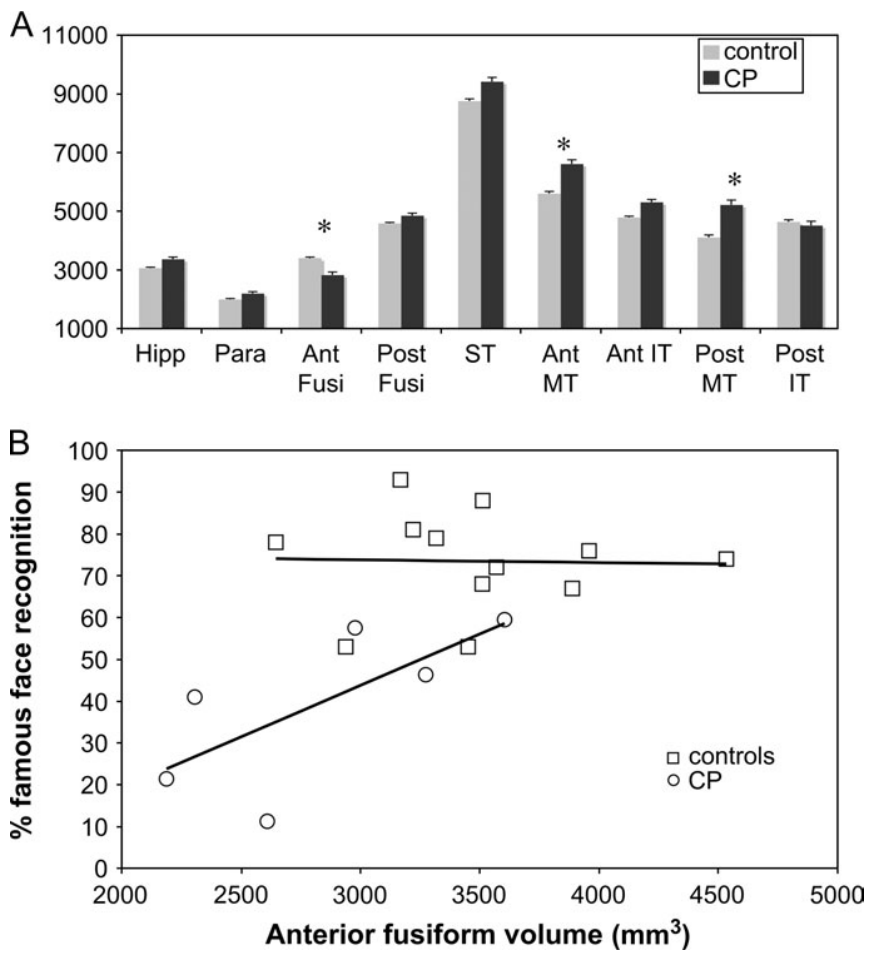

Figure 6. (A) Volumes of temporal lobe structures for 2 groups. Mean (and 1 standard error) volume for the 9 different subregions for the $\mathrm{CP}$ and control individuals. The asterisks denote statistically significant differences, quantified using post hoc Tukey honestly significant differences, at $P<0.05$. (B) Brain-behavior correlation in $\mathrm{CP}$ (circles) and control individuals (squares). Scatter plot of correlation between aF volume and performance on famous faces recognition task for $6 \mathrm{CP}$ individuals and for control subjects.

temporal (MT) gyrus are larger on the right than left (left/right: ant. MT 5952/6255 $\mathrm{mm}^{3}$; post. MT 4332/4991 $\mathrm{mm}^{3}$ ).

Of greater interest, however, are the volumetric differences between the $\mathrm{CP}$ and control group. First, there is a trend, albeit nonsignificant, toward greater temporal lobe volume in the $\mathrm{CP}$ than in the control group $\left(F_{1,16}=3.81, P=0.07\right)$ but this is qualified in an interaction of regional volume by group $\left(F_{8,128}=\right.$ $3.79, P<0.001$ ). (Because the CP individual control subjects were matched on age and gender, we did not consider these as variables in the statistical analysis. Repeating the ANOVAs with these factors as covariates does not change the pattern of the results.) Note that this 2-way interaction does not interact further with side $\left(F_{8,128}=0.75, P>0.5\right)$. Figure $6(A)$ shows the difference in regional volumes for the 2 groups. Post hoc Tukey tests (Bonferroni corrected for multiple comparisons) reveal that the CP group has a larger volume than the controls in the anterior and the posterior portion of the MT gyrus (averaged across both hemispheres). Particularly noteworthy and of great interest for the current purpose, however, is the reduced volume (in an otherwise slightly larger temporal lobe) of the aF gyrus in the CP group compared with the control group (mean, standard deviation: CP $2788.1 \mathrm{~mm}^{3}, 447$; controls 3392.5 $\left.\mathrm{mm}^{3}, 533\right)$. There is no statistical difference between the 2 groups in the volume of any of the other regions.

\section{Functional Significance of Volumetric Differences}

A critical question concerns the relationship between the volumetric alterations and the observed behavioral profile in
CP. If the reduction in the volume of the aF gyrus or the enlargement of the other regions (anterior and posterior MT gyri) is indeed functionally related to the decrement in face processing, then we might expect to find a correlation in the $\mathrm{CP}$ sample between the volumetric measurement and the individual face processing ability. Indeed, a correlation between aF volume (collapsed across side with age partialled out; note that age is included here as this is a within-group assessment) and correct performance on famous face recognition (see Table 1) was obtained in the CP sample (Fig. 6B; Pearson $r^{2}=0.7, P=$ $0.03)$. This correlation is perhaps all the more impressive given that there are only 6 individuals in this sample. We also plot the data from the control individuals in Figure $6(B)$ and there is no correlation between behavior and aF volume in these individuals $(P>0.05)$. This latter finding is perhaps not surprising as there is not much variance in the control subjects in their behavioral performance and the range is thus limited. Note that although there is some apparent overlap between some control subjects and the CP group, the CP individual may be out of the range of his/her own control counterparts (each CP has 2 matched counterparts and this is important given possible effects of age and gender on cortical volume, even if headsize corrected).

The correlation between the aF volume and the ability to discriminate between unknown faces was not significant in the CP individuals $(P=0.8$; for possible reason, see discussion below). There was also no significant correlation with accuracy of face detection, presumably because performance was highly accurate for all CP and there was little variability across the sample. Also, although one might anticipate a similar correlation in the control group, neither the range of behavior nor the range of aF volume varied sufficiently amongst the control participants to permit a reliable evaluation of the brain-behavior correspondence.

In contrast with the positive correlation between aF volume and face recognition in $\mathrm{CP}$, there were no significant (positive or negative) correlations between the enlarged volume in the anterior $\left(r^{2}=0.03, P=0.7\right)$ or posterior $\left(r^{2}=0.49, P>0.1\right)$ portion of the MT gyrus and face processing on any of the 3 tasks (face recognition correlation values provided). The absence of correlations between any of these regions and face recognition raises the speculative possibility that the increased volumes here may perhaps indicate a compensatory response for the aF reduction rather than be directly related to the behavioral profile in CP per se. That the entire temporal lobe volume is not significantly different in the $\mathrm{CP}$ and control groups may suggest that the structural perturbation is in the reduction of the fusiform gyrus with the complement distributed in the temporal gyrus.

A final issue that elucidates the brain-behavior relationship is the consideration of the relationship between the centroid of fusiform activation elicited in these $\mathrm{CP}$ individuals in a previous functional imaging study and the site of the reduced aF volume. One obvious expectation is that, given the apparently normal pattern of BOLD activation evinced by the CP individuals, the volumetric reduction should not overlap with the site of the normal fusiform activation (note that only 4 of the individuals tested here participated in the published functional imaging study by Avidan et al. (2005), but we have the data from the remaining 2 subjects in the identical experiments). We have calculated the coordinates of the peak of face-related fusiform activation across the $6 \mathrm{CP}$ individuals and these are as 
follows: mean $x, y$, and $z$ for right fusiform $37.7,-46.8,-15.8$ and for left fusiform $-43.7,-52,-19.2$, respectively (standard error: right fusiform 1.6, 1.6, 0.95; left fusiform 2.6, 4.5, 1.3). Of great importance is that the centroid of face-related activation falls in the region defined anatomically here as the $\mathrm{pF}$ (the $y$ coordinate for dividing the fusiform anatomically is $y=-30$ ) and there is no significant difference in volume of this $\mathrm{pF}$ region between $\mathrm{CP}$ and controls.

\section{Discussion}

Neurodevelopmental disorders such as developmental dyslexia and dyscalculia as well as CP have long presented a scientific conundrum: In spite of markedly impaired behavior in reading, arithmetic or face recognition, respectively, there has been no clear neural explanation to account for the behavioral alteration in these disorders. Resolving this conundrum has become all the more pressing in light of recent scientific advances suggesting a genetic basis for these disorders and the need to identify potential anatomical structures as the source of these genetic alterations (Fisher 2006; Hulshoff Pol et al. 2006; McGrath et al. 2006). The advent of noninvasive high-resolution structural and fMRI measures has enabled us to begin demarcating the underlying neural substrate in these disorders and has started to shed light on cortical mechanisms subserving brain-behavior relations. In particular, structural measurements of cortical volumes as well as assessment of alterations in tissue density and gray:white matter ratio have proven promising in spite of the tremendous variability in size, shape, and configuration of cortical gyri and sulci across the human brain (Nowinski et al. 1997). In this study, we adopted some of these procedures to examine the structural integrity of the cortex in individuals with CP. Measurements of sulcal depth and distance from the midline were obtained from tracings of the sulci on the inferior temporal surface of structural scans obtained for each individual. Volumetric measurements of temporal cortex as a whole and of multiple subregions of the temporal cortex were also done for each individual through careful parcellation and segregation of the cortical regions.

\section{Anatomical Analysis of Inferotemporal Cortex}

No differences between the $\mathrm{CP}$ and matched control group were observed for sulcal depth or for the deviation from the midline of either of the 2 major inferior temporal sulci (OT and C sulci). The absence of any sulcal differences may, in part, explain why no obvious structural differences are apparent to the naked eye when examining CP structural brain scans. It is also possible that in light of the enormous variability in sulcal patterning in the normal population, discerning any perturbations in the CP population might be extremely difficult.

Despite the absence of group differences in sulcal morphometry, a significant reduction is evident in the volume of the aF gyrus of the CP individuals, relative to their matched counterparts. Importantly, this volumetric diminution is significantly correlated with the face recognition impairment. In contrast with this reduction, there is increased volume in the anterior and posterior MT gyrus, albeit not obviously related to the face recognition per se, and perhaps suggesting a compensatory redistribution of cortical tissue in the $\mathrm{CP}$, compared with the control individuals, whereby total cortical volume is maintained.
No obvious correlation between behavior and the increased MT volume was found.

The finding of a structural difference in the anterior part of the fusiform gyrus of the temporal lobe in CP is particularly interesting given that fMRI studies with these same individuals has not consistently been able to identify any differences in the amplitude, site, or extent of activation in the BOLD pattern of CP compared with matched control participants. It is the case that even in the preeminent face processing area, the fusiform gyrus, there are no apparent group differences in BOLD pattern. This result in itself raises a cautionary note to researchers looking for direct neural correlates of behavior and raises questions concerning the interpretation of BOLD signal and the underlying computations giving rise to this signal (Avidan et al. 2005). What remains puzzling, then, is what underlying mechanism accounts for the behavioral deficit in CP.

In light of the apparently normal BOLD profile in CP along with the anatomical differences reported here, a possible explanation for $\mathrm{CP}$ is that the more posterior regions of the temporal cortex may be operating normally (recall that the centroid of FFA activation is more posterior to the reduced aF volume) but that the failure in face processing arises in the propagation of the output of these more posterior computations to critical anterior face regions, such as the aF gyrus (and perhaps other anterior temporal lobe regions too). It is also possible that feedback from more anterior regions to the $\mathrm{pF}$ cortex also plays a critical role in this more distributed face processing circuit. These results suggest that $\mathrm{CP}$ may be due (perhaps in part) to abnormal development of the anterior portion of the fusiform gyrus.

The proposal that more anterior regions of the temporal lobe are critical for face recognition is also consistent with a number of other recent functional imaging (Bright et al. 2005), single unit physiology (Bussey and Saksida 2005), and computational and neuropsychological (Barense et al. 2005; Lee et al. 2005) studies that have indicated that visual computations are not completed at the more posterior temporal regions. Rather, in the service of more fine-grained discriminations, especially those that support subordinate level identification or individual recognition, more anterior temporal lobe regions are required (Puce et al. 1999; Gobbini and Haxby 2006). It has also been suggested that perceptual processing of complex or highly similar visual stimuli that share features (and faces are a paradigmatic example of this kind of stimulus) relies disproportionately on these more anterior temporal regions (Bussey et al. 2005). In the case of $\mathrm{CP}$, then, although the more posterior regions supporting face recognition may be functional, the restriction potentially arises in the failure to compute more finegrained representations in anterior regions, perhaps as a result of the reduced cortical volume in the aF area. Consequently, there may also be poor propagation of signal to more frontal regions, which are especially engaged by visual stimuli requiring identification (Bar et al. 2006) and which may serve a top-down feedback role (see also Puce et al. 1999), constraining the function of more posterior regions. It is particularly noteworthy that the reduction of volume in this aF area is significantly correlated with the failure to recognize famous faces in the $\mathrm{CP}$ individuals but not the failure to perform face discrimination, a process that does not necessarily rely on face-exemplar identification per se.

One obvious way of examining the anterior portion of the fusiform gyrus more directly would be to conduct neuroimaging 
studies focused on that region. Because of the susceptibility artifact arising from the petrus bone, however, the BOLD signal in fMRI studies in the more anterior parts of the temporal lobe is notoriously weak and difficult to acquire and thus possible group differences in this region may not be detectable. It is the case, however, that novel fMRI procedures are beginning to be able to record BOLD signal in these more anterior temporal regions and, although there is substantial attrition of data given the artifact, procedures for acquiring robust signals are under development. Indeed, in one recent study, face-exemplar information has been evoked in the anterior inferior temporal cortex (Kriegeskorte et al. 2005), consistent with the claim that it is the more anterior temporal regions that are critical for face recognition (Allison et al. 1999) and that the anatomical reduction in this region may underlie CP. The findings we have obtained, along with these other recent studies, may redirect the attention of investigators to this more anterior region as a probable locus for operations that are critical for face identity. If possible, conducting a functional imaging study with the CP individuals to scrutinize the functional integrity of these more anterior regions would be particularly informative.

In addition to scanning more anterior regions, examining the structural connectivity of white matter tracts passing through the fusiform gyrus would be important to document the integrity of the "read out" or propagation of signals to other regions of cortex (as well as feedback signals to this region of cortex). Using diffusion tensor imaging (DTI) and tractography, we have measured the volume and fractional anisotropy (FA) of the 2 major posterior-anterior tracts, the inferior frontooccpital fasciculus and the inferior longitudinal fasciculus, which traverse the FFA (Behrmann et al. 2006). Of great interest is that, relative to the control individuals, these tracts are smaller and there is reduced $\mathrm{FA}$ in the $\mathrm{CP}$. Other cortical tracts, however, show no difference in volume or FA in CP compared with the controls. These findings support the claim that $\mathrm{CP}$ might be mediated by a failure of signal propagation through the distributed face network rather than from a deficiency in the FFA itself.

\section{Alterations in Other Regions of Cortex}

In addition to the alteration in the aF gyrus, the CP individuals showed larger volume than the controls in the MT gyrus. Although we were not able to uncover the functional consequences of this expansion, this enlargement may play a functional, perhaps even compensatory, role. Exploring motion perception of the $\mathrm{CP}$ individuals would be one obvious path to follow with the prediction that their perception of motion might be more fine-grained than that of their control counterparts. Although one might predict reorganization in sensory cortex to have obvious functional correlates, as we suggest, this need not always be directly the case. For example, deaf individuals do show reasonably obvious changes in multisensory cortex and although there has long been the clear prediction that they would show enhanced visual performance, this is not always so (Bavelier et al. 2006). To the extent that behavioral changes are evident in deaf individuals, these are not widespread but appear in selective aspects of vision that are attentionally demanding and would normally benefit from auditory-visual convergence. Thus, although one might expect changes in cortical organization to be mirrored in behavioral alterations and vice versa, the correspondence is not so obvious. It is also the case that we do not yet have a good neurobiological explanation for the histological source of any of these volumetric cortical changes. The changes might reflect increase in spacing between neurons, changes in neuronal soma size, or some combination thereof and these may have different structural as well as functional consequences. Clearly, these are directions for future research and data from such investigations will surely inform our understanding of brain-behavior correspondences.

The findings thus far have mostly concentrated on the aF and the MT gyrus as these were the only 2 clearly differentiable regions across the $\mathrm{CP}$ and controls. We observed no difference in amygdala size in the CP individuals. Note, however, that the amygdala was measured in concert with the parahippocampus (as they are difficult to segregate anatomically). Given the importance of the amygdala in emotional processing, further attention to its structure might be warranted in CP. We do note though that, in one of our previous studies (Humphreys et al. in press), the $\mathrm{CP}$ individuals performed equivalently to the matched controls on emotional expression discrimination but other reports of CP individuals do report deficits in expression recognition (Kress and Daum 2003a). Further attention to this issue is clearly warranted.

\section{Conclusion}

In conclusion, these findings identify, for the first time, a candidate cortical region that might serve as the neural origin of CP. Not only does this brain-behavior correspondence potentially uncover key cognitive and neural substrates in this neurodevelopmental disorder but it also suggests a critical role for the aF region of the temporal lobe in face processing more generally. Whether the reduced volume is a consequence of alteration in white matter tracts in this area (perhaps reduced myelination), as we have suggested from the DTI findings, and/ or of decreased gray matter volume remains to be definitively determined. Also, whether these findings apply to all individuals with CP, given the reports of heterogeneity within this population (Kress and Daum 2003a; Le Grand et al. 2006), also remains to be assessed. This brain-behavior analysis in $\mathrm{CP}$, afforded by the advances in high-resolution human neuroimaging, may also serve as a model for uncovering similar correspondences in other, cognitively circumscribed neurodevelopmental disorders. Furthermore, given that most, if not all, CP individuals have affected family members (Kennerknecht et al. 2006), these results highlight a possible genetically specified perturbation of cortex that warrants further targeted investigation.

\section{Notes}

The authors thank Linda Moya and Grace Leonard Lee for assistance in data collection, Cibu Thomas for useful discussion and Scott Kurdilla, Kwan Jin-Jung, and Debbie Vizlay of the Brain Imaging Research Center in Pittsburgh, PA, for their invaluable assistance. Thanks to Shahryar Rafi-Tari and Gregory Szilagyi for their contribution to the data analysis and investigation. This research was funded by a grant to Marlene Behrmann from the National Institute of Mental Health (MH54246). Conflict of Interest: None declared.

Address correspondence to Marlene Behrmann, Department of Psychology, Carnegie Mellon University, Pittsburgh, PA 15213-3890, USA. Email: behrmann@cmu.edu. 
Appendix 1

Normalized (raw distance/maximal head width) from midline for $\mathrm{C}$ and OT sulci for each $\mathrm{CP}$ and each control individual as well as the group means

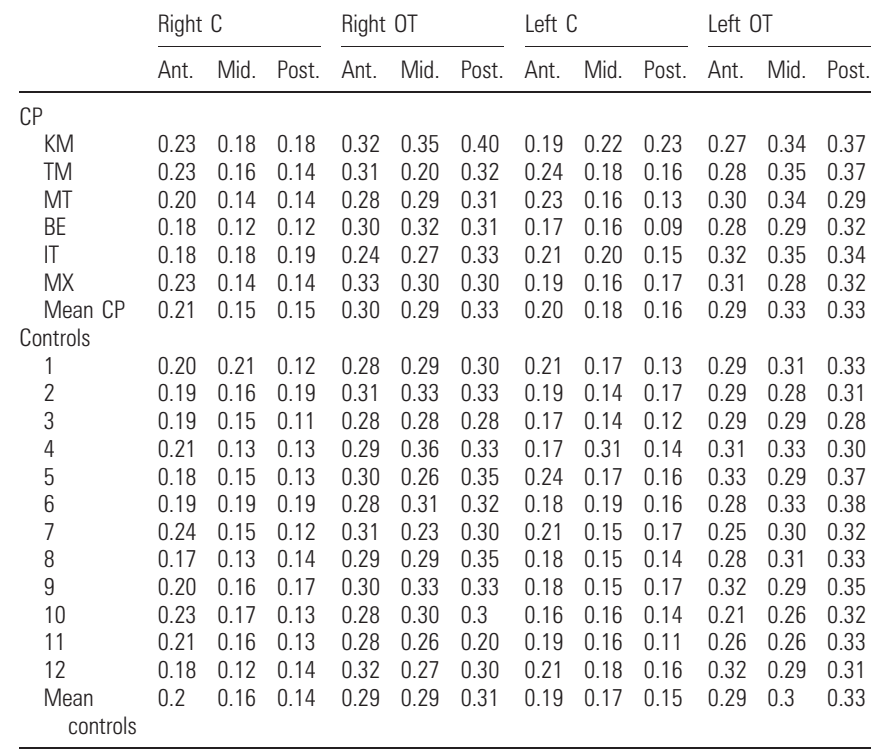

Sulcal depth for $\mathrm{C}$ and $\mathrm{OT}$ sulci for each $\mathrm{CP}$ and each control individual as well as the group means

\begin{tabular}{|c|c|c|c|c|c|c|c|c|}
\hline & \multicolumn{2}{|l|}{ Right } & \multicolumn{2}{|l|}{ Left } & \multicolumn{2}{|l|}{ Right } & \multicolumn{2}{|l|}{ Left } \\
\hline & $\begin{array}{l}\text { C } \\
\text { Ant. }\end{array}$ & $\begin{array}{l}\text { OT } \\
\text { Mid. }\end{array}$ & $\begin{array}{l}\text { C } \\
\text { Ant. }\end{array}$ & OT & C & $\begin{array}{l}\text { OT } \\
\text { Mid. }\end{array}$ & $\begin{array}{l}\text { C } \\
\text { Post. }\end{array}$ & $\begin{array}{l}\text { OT } \\
\text { Mid. }\end{array}$ \\
\hline \multicolumn{9}{|l|}{$\mathrm{CP}$} \\
\hline KM & 6.17 & 12.71 & 6.32 & 13.05 & 12.11 & 13.14 & 10.24 & 11.38 \\
\hline TM & 10.06 & 8.45 & 10.89 & 13.73 & 13.18 & 11.05 & 9.72 & 15.99 \\
\hline MT & 10.99 & 11.35 & 9.34 & 8.82 & 11.26 & 12.69 & 13.08 & 16.74 \\
\hline $\mathrm{BE}$ & 8.22 & 11.51 & 8.23 & 11.34 & 15.17 & 12.25 & 14.44 & 13.7 \\
\hline IT & 6.77 & 9.34 & 8.78 & 9.11 & 9.08 & 11.72 & 10.06 & 9.35 \\
\hline$M X$ & 7.61 & 13.94 & 8.37 & 12.42 & 10.68 & 11.71 & 10.08 & 14.06 \\
\hline Mean CP & 8.3 & 11.2 & 8.66 & 11.4 & 11.9 & 12.1 & 11.3 & 13.5 \\
\hline \multicolumn{9}{|l|}{ Controls } \\
\hline 1 & 9.2 & 12.96 & 14.27 & 12.76 & 14.79 & 12.34 & 11.75 & 11.78 \\
\hline 2 & 9.63 & 13.47 & 9.22 & 12.38 & 11.42 & 16.12 & 14.19 & 14.71 \\
\hline 3 & 7.4 & 12.62 & 8.89 & 10.84 & 13.97 & 13.73 & 10.52 & 14.81 \\
\hline 4 & 10.62 & 13.85 & 10.4 & 11.06 & 13.55 & 10.73 & 13.14 & 10.52 \\
\hline 5 & 8.2 & 14.44 & 5.68 & 11.48 & 13.8 & 12.53 & 12.15 & 13.64 \\
\hline 6 & 11.26 & 14.96 & 17.8 & 9.43 & 12.52 & 11.91 & 9.24 & 14.38 \\
\hline 7 & 11.14 & 12.42 & 8.94 & 11.79 & 11.28 & 12.28 & 11.92 & 14.73 \\
\hline 8 & 15.25 & 10.59 & 15.91 & 5.97 & 12.24 & 12.52 & 13.59 & 10.46 \\
\hline 9 & 13.18 & 7.44 & 14.15 & 7.73 & 11.7 & 11.75 & 12.39 & 15.65 \\
\hline 10 & 9.78 & 12.7 & 10.56 & 9.14 & 12.45 & 12.14 & 12.64 & 8.73 \\
\hline 11 & 7.75 & 9.29 & 8.48 & 10.68 & 12.49 & 11.81 & 13.02 & 12.03 \\
\hline 12 & 8.95 & 10.91 & 6.46 & 8.82 & 11.95 & 7.96 & 10.7 & 12.39 \\
\hline Mean controls & 10.2 & 12.1 & 10.9 & 10.2 & 12.7 & 12.2 & 12.1 & 12.8 \\
\hline
\end{tabular}

\section{References}

Allison T, Puce A, Spencer DD, McCarthy G. 1999. Electrophysiological studies of human face perception. I: Potentials generated in occipitotemporal cortex by face and non-face stimuli. Cereb Cortex. 9:415-430

ANALYZE. 2004. ANALYZE AVW ${ }^{\top M}$ Software. Mayo Foundation. Rochester, MN: Biomedical Imaging Resource.

Avidan G, Hasson U, Malach R, Behrmann M. 2005. Detailed exploration of face-related processing in congenital prosopagnosia: 2. Functional neuroimaging findings. J Cogn Neurosci. 17:1150-1167.

Avidan G, Thomas C, Behrmann M. Forthcoming. An integrative approach towards understanding the psychological and neural basis of congenital prosopagnosia. In: Harris L, Jenkin M, editors. Cortical mechanisms of vision.

Bar M, Kassam KS, Ghuman AS, Boshyan J, Schmid AM, Dale AM, Hamalainen MS, Marinkovic K, Schacter DL, Rosen BR, et al. 2006. Top-down facilitation of visual recognition. Proc Natl Acad Sci USA. 103:449-454.

Barense M, Bussey TJ, Lee ACH, Rogers TT, Davies RR, Saksida LM, Murray EA, Graham KS. 2005. Functional specialization in the human medial temporal lobe. J Neurosci. 25:10239-10246.

Barton JJ, Cherkasova MV, Press DZ, Intriligator JM, O'Connor M. 2004. Perceptual functions in prosopagnosia. Perception. 33:939-956.

Barton JJS. 2003. Disorders of face perception and recognition. Neurol Clin North Am. 21:521-548.

Bavelier D, Dye MW, Hauser PC. 2006. Do deaf individuals see better? Trends Cogn Sci. 10:512-518.

Behrmann M, Avidan G. 2005. Congenital prosopagnosia: face-blind from birth. Trends Cogn Sci. 9:180-187.

Behrmann M, Avidan G, Humphreys K, Thomas C. Forthcoming. Congenital and acquired prosopagnosia: flip sides of the same coin? In: Gauthier I, Bub D, Tarr M, editors. Perceptual expertise: bridging brain and behavior. New York: Oxford University Press.

Behrmann M, Avidan G, Marotta JJ, Kimchi R. 2005. Detailed exploration of face-related processing in congenital prosopagnosia: 1. Behavioral findings. J Cogn Neurosci. 17:1130-1149.

Behrmann M, Thomas C, Avidan G, Jung KJ. 2006. Connectivity alterations in ventral visual cortex may explain congenital prosopagnosia. Society for Neurosciences annual meeting, Atlanta, October.

Belmonte MK, Allen G, Beckel-Mitchener A, Boulanger LM, Carper RA, Webb SJ. 2004. Autism and abnormal development of brain connectivity. J Neurosci. 24:9228-9231.

Bentin S, Deouell L. 2000. Structural encoding and identification in face processing: ERP evidence for separate mechanisms. Cogn Neuropsychol. 17:35-54.

Bentin S, Deouell LY, Soroker N. 1999. Selective visual streaming in face recognition: evidence from developmental prosopagnosia. Neuroreport. 10:823-827.

Bouvier SE, Engel SA. 2006. Behavioral deficits and cortical damage loci in cerebral achromatopsia. Cereb Cortex. 16:183-191.

Bright P, Moss HE, Stamatakis EA, Tyler LK. 2005. The anatomy of object processing: the role of anteromedial temporal cortex. Q J Exp Psychol B. 58:361-377.

Brown WE, Eliez S, Menon V, Rumsey JM, White CD, Reiss AL. 2001. Preliminary evidence of widespread morphological variations of the brain in dyslexia. Neurology. 56:781-783.

Bussey TJ, Saksida LM. 2005. Object memory and perception in the medial temporal lobe: an alternative approach. Curr Opin Neurobiol. 15:730-737.

Bussey TJ, Saksida LM, Murray EA. 2005. The perceptual-mnemonic/ feature conjunction model of perirhinal cortex function. Q J Exp Psychol. 58B:269-282.

Callen DJ, Black SE, Gao F, Caldwell CB, Szalai JP. 2001. Beyond the hippocampus: MRI volumetry confirms widespread limbic atrophy in AD. Neurology. 57:1669-1674.

Casanova MF, Christensen JD, Giedd J, Rumsey JM, Garver DL, Postel GC. 2005. Magnetic resonance imaging study of brain asymmetries in dyslexic patients. J Child Neurol. 20:842-847.

Damasio A, Damasio H. 1980. Prosopagnosia: behavioral and anatomical aspects. Neurology. 30:390-391 [abstract].

Damasio A, Damasio H, Tranel D. 1986. Prosopagnosia: anatomic and physiological aspects. In: Ellis HD, Jeeves MA, Newcombe F, Young A, editors. Aspects of face processing. Dordrecht: Martinus Nijhoff. p. 279-290.

de Gelder B, Stekelenburg JJ. 2005. Naso-temporal asymmetry of the $\mathrm{N} 170$ for processing faces in normal viewers but not in developmental prosopagnosia. Neurosci Lett. 376:40-45.

De Haan EH. 1999. A familial factor in the development of face recognition deficits. J Clin Exp Neuropsychol. 21:312-315.

De Renzi E. 1986. Current issues in prosopagnosia. In: Ellis H, Jeeves MA, Newcombe F, Young AW, editors. Aspects of face processing. Dordrecht: Martinus Nijhoff. 
De Renzi E, Perani D, Carlesimo GA, Silveri MC, Fazio F. 1994 Prosopagnosia can be associated with damage confined to the right hemisphere-an MRI and PET study and a review of the literature. Neuropsychologia. 32:893-902.

Dorsaint-Pierre R, Penhune VB, Watkins KE, Neelin P, Lerch JP, Bouffard M, Zatorre RJ. 2006. Asymmetries of the planum temporale and Heschl's gyrus: relationship to language lateralization. Brain. 129:1164-1176.

Duchaine B, Nakayama K. 2005. Dissociations of face and object recognition in developmental prosopagnosia. J Cogn Neurosci. 17:249-261.

Duchaine B, Yovel G, Butterworth EJ, Nakayama K. 2006. Prosopagnosia as an impairment to face-specific mechanisms: elimination of the alternative hypotheses in a developmental case. Cogn Neuropsychol. 23:714-747.

Duchaine BC, Nakayama K. 2006. Developmental prosopagnosia: a window to content-specific face processing. Curr Opin Neurobiol. 16:166-173.

Eliez S, Rumsey JM, Giedd JN, Schmitt JE, Patwardhan AJ, Reiss AL. 2000 Morphological alteration of temporal lobe gray matter in dyslexia: an MRI study. J Child Psychol Psychiatry. 41:637-644.

Farah MJ. 2004. Visual agnosia. Cambridge, MA: MIT Press.

Fisher SE. 2006. Tangled webs: tracing the connections between genes and cognition. Cognition. 101:270-279.

Gobbini MI, Haxby JV. 2007. Neural systems for recognition of familiar faces. Neuropsychologia. 45:32-41.

Grueter M, Grueter T, Bell V, Horst J, Laskowski W, Sperling K, Halligan PW, Ellis HD, Kennerknecht I. Forthcoming 2007. Hereditary prosopagnosia: the first case series. Cortex.

Hadjikhani N, De Gelder B. 2002. Neural basis of prosopagnosia. Hum Brain Mapp. 16:176-182.

Harris AM, Duchaine BC, Nakayama K. 2005. Normal and abnormal face selectivity of the M170 response in developmental prosopagnosics. Neuropsychologia. 43:2125-2136.

Hasson U, Avidan G, Deouell LY, Bentin S, Malach R. 2003. Face-selective activation in a congenital prosopagnosic subject. J Cogn Neurosci. 15:419-431.

Hulshoff Pol HE, Schnack HG, Posthuma D, Mandl RC, Baare WF, van Oel C, van Haren NE, Collins DL, Evans AC, Amunts K, et al. 2006. Genetic contributions to human brain morphology and intelligence. J Neurosci. 26:10235-10242

Humphreys K, Avidan G, Behrmann M. 2007. A detailed investigation of facial expression processing in congenital prosopagnosia as compared to acquired prosopagnosia. Exp Brain Res. 176:356-373.

Jones RD, Tranel D. 2001. Severe developmental prosopagnosia in a child with superior intellect. J Clin Exp Neuropsychol. 23: 265-273.

Kanwisher N, McDermott J, Chun MM. 1997. The fusiform face area a module in human extrastriate cortex specialized for face perception. J Neurosci. 17:4302-4311.

Kennerknecht I, Grueter T, Welling B, Wentzek S, Horst J, Edwards S, Grueter M. 2006. First report of prevalence of non-syndromic hereditary prosopagnosia (HPA). Am J Med Genet A. 140:1617-1622.

Kleinschmidt A, Cohen L. 2006. The neural bases of prosopagnosia and pure alexia: recent insights from functional neuroimaging. Curr Opin Neurol. 19:386-391.

Kress T, Daum I. 2003a. Developmental prosopagnosia: a review. Behav Neurol. 14:109-121.

Kress T, Daum I. 2003b. Event-related potentials reflect impaired face recognition in patients with congenital prosopagnosia. Neurosci Lett. 352:133-136.
Kriegeskorte N, Formisano E, Sorger B, Goebel R. 2005. Individual faces elicit distinct fMRI response patterns in human anterior temporal cortex. Society for Neuroscience, Washington (DC).

Laakso MP, Partanen K, Riekkinen P, Lehtovirta M, Helkala EL, Hallikainen M, Hanninen T, Vainio P, Soininen H. 1996. Hippocampal volumes in Alzheimer's disease, Parkinson's disease with and without dementia, and in vascular dementia: an MRI study. Neurology. 46:678-681.

Le Grand R, Cooper PA, Mondloch CJ, Lewis TL, Sagiv N, de Gelder B, Maurer D. 2006. What aspects of face processing are impaired in developmental prosopagnosia? Brain Cogn. 61:139-58.

Lee AC, Bussey TJ, Murray EA, Saksida LM, Epstein RA, Kapur N, Hodges JR, Graham KS. 2005. Perceptual deficits in amnesia: challenging the medial temporal lobe 'mnemonic' view. Neuropsychologia. 43:1-11.

Lee CU, Shenton M, Salisbury DF, Kasai K, Onitsuka T, Dickey CC, Yurgelun-Todd D, Kikinis R, Jolesz FA, McCarley RW. 2002. Fusiform gyrus volume reduction in first-episode schizophrenia. Arch Gen Psychiatry. 59:775-781.

Levitt JG, Blanton RE, Smalley S, Thompson PM, Guthrie D, McCracken JT, Sadoun T, Heinichen L, Toga AW. 2003. Cortical sulcal maps in autism. Cereb Cortex. 13:728-735.

McGrath LM, Smith SD, Pennington BF. 2006. Breakthroughs in the search for dyslexia candidate genes. Trends Mol Med. 12:333-341.

Molko N, Cachia A, Riviere D, Mangin JF, Bruandet M, Le Bihan D, Cohen L, Dehaene S. 2003. Functional and structural alterations of the intraparietal sulcus in a developmental dyscalculia of genetic origin. Neuron. 40:847-858.

Nowinski WL, Fang A, Nguyen BT, Raphel JK, Jagannathan L, Raghavan R, Bryan RN, Miller GA. 1997. Multiple brain atlas database and atlasbased neuroimaging system. Comput Aided Surg. 2:42-66.

Nunn JA, Postma P, Pearson R. 2001. Developmental prosopagnosia: should it be taken at face value? Neurocase. 7:15-27.

Oldfield RC. 1971. The assessment and analysis of handedness: the Edinburgh inventory. Neuropsychologia. 9:97-113.

Puce A, Allison T, McCarthy G. 1999. Electrophysiological studies of human face perception. III: effects of top-down processing on facespecific potentials. Cereb Cortex. 9:445-458.

Reiss AL, Eckert MA, Rose FE, Krachemskiy A, Kesler S, Chang M, Reynolds MF, Kwon H, Galaburda A. 2004. An experiment of nature: brain anatomy parallels cognition and behavior in Williams syndrome. J Neurosci. 24:5009-5015.

Rettmann ME, Kraut MA, Prince JL, Resnick SM. 2005. Cross-sectional and longitudinal analyses of anatomical sulcal changes associated with aging. Cereb Cortex. 16:1584-94.

Sergent J, Signoret JL. 1992. Functional and anatomical decomposition of face processing: evidence from prosopagnosia and PET study of normal subjects. Philos Trans R Soc Lond B Biol Sci. 335:55-61; discussion 61-52.

Vidal CN, Rapoport JL, Hayashi KM, Geaga JA, Sui Y, McLemore LE, Alaghband Y, Giedd JN, Gochman P, Blumenthal J, et al. 2006. Dynamically spreading frontal and cingulate deficits mapped in adolescents with schizophrenia. Arch Gen Psychiatry. 63:25-34.

Vinckenbosch E, Robichon F, Eliez S. 2005. Gray matter alteration in dyslexia: converging evidence from volumetric and voxel-by-voxel MRI analyses. Neuropsychologia. 43:324-331.

von Kriegstein K, Kleinschmidt A, Giraud AL. 2006. Voice recognition and cross-modal responses to familiar speakers' voices in prosopagnosia. Cereb Cortex. 16:1314-1322.

Yovel G, Duchaine B. 2006. Specialized face perception mechanisms extract both part and spacing information: evidence from developmental prosopagnosia. J Cogn Neurosci. 18:580-593. 